\title{
Diferenças de rendimento do trabalho de homens e mulheres com educação superior nas metrópoles ${ }^{\star}$
}

\author{
Eugenia Troncoso Leone** \\ Paulo Baltar ${ }^{* *}$
}

\begin{abstract}
Este artigo faz uma análise das diferenças de rendimento entre homens e mulheres ocupados, com nível superior de escolaridade, no conjunto das regiões metropolitanas do país, partindo-se de duas constatações: a) as diferenças de rendimento entre homens e mulheres, apesar de terem diminuído na última década, continuam grandes e fazem parte de um contexto de enorme diferenciação de remunerações, em que sobressaem as diferenças de escolaridade, destacando-se os altos rendimentos do nível superior, cujas diferenças em relação aos outros níveis de escolaridade ampliaram-se ainda mais nesta década e b) as maiores diferenças de rendimento entre homens e mulheres ocorrem exatamente no nível superior de escolaridade, justamente naqueles em que as mulheres têm maior participação. $O$ artigo analisa as mudanças ocorridas na década de 90, no nível e dispersão dos rendimentos dos ocupados, mostrando as diferenças segundo sexo, escolaridade e posição na ocupação. Também é feito um estudo específico, para o ano de 2004, das diferenças de rendimento entre homens mulheres com curso superior, conforme posição na ocupação e setor de atividade. É considerado, ainda, o tipo de ocupação (dirigentes em geral, profissionais das ciências e artes, técnicos de nível médio, trabalhadores de serviços administrativos, outras ocupações) como "proxy" da hierarquia dos cargos. A fonte de dados utilizada é a Pesquisa Nacional por Amostra de Domicílios $-P N A D$.
\end{abstract}

Palavras-chave: Mercado de trabalho. Rendimentos. Gênero. Escolaridade.

\section{Introdução}

A crescente participação das mulheres na atividade econômica e seu maior peso no conjunto da força de trabalho não conseguiram reverter ainda a enorme desigualdade de gênero no trabalho remunerado, que se manifesta em salários inferiores, maior desemprego, maior informalização do trabalho, ocupações de menor prestígio social e menos direitos trabalhistas e previdenciários.
A continuidade das obrigações e responsabilidades das mulheres na família, não se alterando substancialmente a repartição entre sexos dos afazeres domésticos e sem o apoio necessário de serviços públicos especializados, não impediu a consolidação da participação das mulheres na atividade econômica, mas levou a estratégias individuais de adaptação que não evitaram as repercussões negativas sobre a família. Além disso, tais estratégias, ao dependerem do nível socioeconômico das

\footnotetext{
*Trabalho apresentado no XV Encontro Nacional de Estudos Populacionais, da Abep, realizado em Caxambu-MG, de 20 a 24 de setembro de 2006.

** Professores do Instituto de Economia da Universidade Estadual de Campinas (Unicamp) e pesquisadores do Centro de Estudos Sindicais e Economia do Trabalho (Cesit).
} 
pessoas, tiveram implicações especiais no sentido de reforçar aspectos importantes da desigualdade do trabalho remunerado.

$O$ acesso maciço e permanente das mulheres à atividade econômica, em um modelo de trabalho em que o homem tem disponibilidade absoluta para dedicar-se ao trabalho remunerado, provoca, de um lado, grandes diferenças entre homens e mulheres e, de outro, enorme diferenciação entre as próprias mulheres, reproduzindo as diferenças da sociedade (CARRASCO et al. 1998). A força de trabalho feminina se polariza: em um extremo, encontra-se um reduzido grupo de mulheres com alto nível de estudos e qualificação, que se incorporam em trabalhos estáveis e de prestígio social, trabalhando jornada completa no setor público ou em grandes empresas; no outro extremo, tem-se a grande maioria das mulheres em condições de trabalho mais vulneráveis devido a ocupações com menor poder de negociação, menor qualificação, temporárias ou em tempo parcial, em pequenas empresas do setor privado e/ou atividades consideradas socialmente femininas (VARELLA, 2001).

As mulheres trabalhadoras constituem, assim, um grupo heterogêneo, marcado pelas diferenças econômicas e sociais existentes na sociedade da qual formam parte, o que não somente afeta o acesso às ocupações, mas também está presente na hora de determinar os recursos materiais e as estratégias pessoais disponíveis para enfrentar os condicionantes derivados das responsabilidades atribuídas a seu gênero, na hora de optar pelo trabalho remunerado (VARELLA, 2001).

Esses efeitos sobre a desigualdade do trabalho remunerado e as dificuldades da mudança do papel da mulher na família e na atividade econômica, com a continuidade do modelo do comportamento masculino e na ausência de apoio de serviços públicos especializados, foram acentuados pelas alterações que ocorreram, ao mesmo tempo, na economia e no mercado de trabalho do país.

De fato, nos anos 90, a insuficiente ampliação do mercado de trabalho manifestou-se na queda da participação dos jovens, afetando principalmente os homens que tinham uma alta taxa de participação, e no aumento do desemprego da população adulta, atingindo principalmente as mulheres, que elevaram a taxa de participação (LAVINAS, 2000). O maior aumento do desemprego feminino reflete a ampliação da participação das mulheres na atividade econômica, enquanto a taxa de desemprego masculina não teve crescimento tão acentuado, como conseqüência da diminuição da participação dos homens no mercado de trabalho, devido à desistência por desalento na busca de um trabalho remunerado.

Mas o crescimento de oportunidades de ocupação para a população ativa não somente foi insuficiente como também passou por profundas modificações no perfil setorial e por posição na ocupação. Assim, ao longo da década de 90 , diminuiu a geração de oportunidades de ocupação na produção de bens e aumentou no comércio e na prestação de serviços. De forma simultânea, reduziu-se o peso do emprego assalariado em estabelecimentos e ampliaram-se a ocupação por conta-própria e o trabalho doméstico remunerado. 0 decréscimo do emprego assalariado em estabelecimentos ocorreu com diminuição da participação da grande empresa e aumento das pequenas, elevando a proporção dos empregados assalariados de estabelecimentos sem contrato de trabalho formalizado na carteira profissional (BALTAR, 2003 e 2005).

Mudanças na estruturação da economia, com pouca ampliação da produção, significaram muita eliminação de empregos comparativamente aos novos postos de trabalho criados. Esta situação do mercado de trabalho tende a afetar desfavoravelmente e mais intensamente os grupos de trabalhadores que tinham melhor posição no passado. Alguns dos melhores postos de trabalho foram eliminados, ou pelo menos muito modificados, obrigando os antigos empregados a disputarem as novas posições com os que estão entrando no mercado de trabalho, sendo que, dependendo da natureza dos postos de trabalho, antigos e novos, a experiência prévia 
freqüentemente signifique uma desvantagem para a competição.

As mudanças na estruturação produtiva que afetaram significativamente o mercado de trabalho brasileiro obrigaram homens $e$ mulheres, jovens e adultos a se adaptarem de forma diferenciada. O crescimento contínuo e sustentado da participação da mulher no mercado de trabalho, em particular das esposas com filhos (MONTALI, 2004), parece indicar uma situação mais favorável para as mulheres do que para os homens no que se refere à expansão do seu nível de ocupação. Conforme Lavinas (2002), a maior flexibilização do mercado de trabalho e a precarização das relações de emprego têm favorecido as mulheres, aumentando sua empregabilidade, como conseqüência de sua melhor adaptação às tendências atuais do trabalho.

Assim, ocupações em tempo parcial atraem mais mulheres, pois Ihes permitem conciliar melhor o trabalho doméstico com o remunerado, ainda quando seja aceitando remunerações inferiores, mesmo tendo deixado a condição de mão-de-obra secundária. Mas esses contratos flexíveis significam uma perda de direitos sociais conquistados há muito tempo pelos trabalhadores, como férias, seguro-desemprego e licença maternidade (HIRATA, 2001).

Outros aspectos que reforçariam a empregabilidade das mulheres provêm de características e capacidades atribuídas a seu sexo, advindas do velho padrão de divisão sexual do trabalho, que se transformaram em vantagens profissionais, como melhor desenvoltura em atividades de atendimento personalizado a públicos diferenciados (LAVINAS, 2002; GUEDES e ALVES, 2004).

Assim, a expansão da atividade feminina não tem sido acompanhada por melhora de direitos e oportunidades e as mulheres ainda estão longe de desfrutar posições equitativas em relação aos homens no mercado de trabalho. A educação superior, entretanto, tem sido um fator importante no progresso profissional das mulheres, principalmente no setor público e nas grandes organizações do setor privado, onde o recrutamento e a seleção são muito mais marcados pelas formalidades dos processos burocráticos.
Conforme Beltrão e Alves (2004), as conquistas femininas na educação vêm acontecendo de maneira progressiva e, no que se refere ao nível superior, a proporção de mulheres de coortes mais jovens que concluem o curso superior é quase duas vezes a dos homens.

Entretanto, conforme os mesmos autores, essas conquistas femininas no campo educacional não foram acompanhadas por conquistas de dimensão equivalente no mercado de trabalho. De fato, os maiores níveis de escolaridade das mulheres ocupadas não lhes têm garantido o acesso a níveis de rendimento semelhantes aos masculinos. As diferenças de rendimento entre os sexos, embora ocorram em todos os níveis de escolaridade, são maiores na educação superior, em que as mulheres constituem mais da metade dos ocupados com essa escolaridade. Tudo indica que existem muitas ocupações de nível superior de escolaridade para mulheres que não proporcionam rendimentos tão elevados como algumas ocupações de mesmo grau de instrução para homens, além da maior dificuldade de ascensão por parte das mulheres aos cargos de chefia (LEONE e BALTAR, 2005).

Este artigo tem por objetivo estudar as diferenças de rendimento do trabalho entre homens e mulheres com nível superior de escolaridade, no conjunto das dez principais regiões metropolitanas do país: Belém, Fortaleza, Recife, Salvador, Rio de Janeiro, Belo Horizonte, Curitiba, Porto Alegre, São Paulo e Distrito Federal. A fonte de dados utilizada é a Pesquisa Nacional por Amostra de Domicílios - PNAD, referente a 2004. Utilizou-se o conceito restrito de ocupação, excluindo-se os dados relativos a autoconstrução, autoconsumo e trabalho nãoremunerado com duração semanal inferior a 15 horas. Os rendimentos do trabalho foram expressos em salários mínimos/hora. $\mathrm{Na}$ análise das diferenças de rendimento, usou-se a mediana da distribuição dos rendimentos.

Além desta introdução, o artigo apresenta uma descrição da evolução das diferenças de rendimento e escolaridade entre homens e mulheres na década de 90 . 
Também são analisadas as diferenças de rendimentos do trabalho, em 2004, segundo posição na ocupação e escolaridade. Finalmente, são estudadas as diferenças por sexo dos rendimentos das pessoas ocupadas com nível superior de instrução, incorporando na análise o tipo de ocupação e o setor de atividade.

\section{Diferenças de rendimento e escolaridade entre homens e mulheres na década de $90^{1}$}

A principal manifestação da consolidação da mulher no mercado de trabalho se revela na constante ampliação das taxas de participação feminina, em todas as idades, mas principalmente entre as mais adultas e casadas, elevando assim o peso da mulher na força de trabalho. No Brasil metropolitano, as mulheres passaram a representar, em 2002, 44,5\% da força de trabalho das metrópoles e $43 \%$ da parcela da PEA que se encontrava ocupada. Devese ressaltar, entretanto, que o fato de as mulheres serem $54 \%$ dos desempregados revela a insuficiência da geração de postos de trabalho pela atividade econômica para absorção da totalidade do crescimento da PEA feminina.

A consolidação da participação feminina em um mercado de trabalho desfavorável para a população em geral, e para a mulher em particular, dificultou uma maior diversificação da inserção da mulher por setor de atividade econômica e tipo de ocupação e a obrigou a continuar muito concentrada em determinados tipos de ocupação e setores de atividade, destacando-se alguns postos de trabalho menos organizados, menos formalizados e pior remunerados. $O$ fraco desempenho do mercado de trabalho, na década de 90 , repercutiu negativamente na estrutura de posições na ocupação, ressaltando-se os aumentos expressivos do emprego não-formal em estabelecimento, do emprego doméstico e do trabalho por conta-própria. A categoria dos empregadores, embora tenha uma participação muito pequena na ocupação total, registrou expressivo aumento proporcional na década, refletindo a proliferação de pequenas empresas reforçada pelo fenômeno da terceirização, tendo sido acompanhada da elevação da proporção de empregados sem carteira assinada no mercado de trabalho.

Um fato curioso dos anos 90 foi o aumento dos rendimentos do trabalho exatamente nas categorias ocupacionais que mais cresceram na década, isto é, no emprego não-formal, no emprego doméstico e no trabalho por conta-própria. Mas o rendimento médio dos trabalhadores elevou-se devido, principalmente, ao expressivo aumento do rendimento médio dos empregadores. $\mathrm{O}$ crescimento dos rendimentos dos assalariados de estabelecimento sem vínculo formal de trabalho, dos trabalhadores domésticos e por contaprópria contribuiu muito pouco para a expansão do rendimento médio dos ocupados, devido ao baixo nível do rendimento auferido por esses trabalhadores.

$\mathrm{O}$ aumento do rendimento médio do trabalho, entretanto, não expressa devidamente a evolução do nível desses rendimentos. $\mathrm{O}$ crescimento muito menor da mediana dos rendimentos exprime melhor essa evolução, traduzindo os enormes aumentos do emprego doméstico, daquele em estabelecimentos sem carteira de trabalho e de trabalhadores por conta-própria, que continuam muito mal remunerados, embora tenham apresentado ligeira elevação de suas remunerações medianas. Neste particular, destaca-se o intenso crescimento do emprego doméstico, na década, que reverteu tendência anterior de redução deste tipo de atividade, observando-se, inclusive, uma relativa estruturação desta ocupação, elevando-se a proporção de trabalhadoras com carteira de trabalho, além de uma notória melhoria de suas remunerações, inde-

${ }^{1}$ Este item baseia-se no artigo Mercado de trabalho metropolitano: gênero e diferenças de rendimento, 1992-2002, apresentado no IX Encontro Nacional de Estudos do Trabalho, promovido pela Associação Brasileira de Estudos do Trabalho - Abet, realizado em Recife em outubro de 2005. 
pendentemente da pos-se da carteira de trabalho (MELO, 1999).

A relativa estruturação do emprego doméstico foi parte de um processo mais geral, que atingiu também outras ocupações tradicionalmente mal remuneradas. Essas ocupações eram portas de passagem para jovens com desvantagem na disputa pelo primeiro emprego, que se deslocavam para outras ocupações tentando consolidar sua participação na atividade econômica, mas que agora, por falta de alternativas de emprego, permanecem nas mesmas ocupações mal remuneradas, ampliando a fração de pessoas adultas. Esse aumento da proporção de adultos nas ocupações mal remuneradas ampliou o segmento de ocupados com rendimento pouco maior que o salário mínimo, embora nessas ocupações também tenha aumentado o número absoluto de pessoas ganhando menos que o salário mínimo.

Refletindo as mudanças no perfil da distribuição das pessoas por faixas de salário mínimo nas ocupações mal remuneradas, diminuíram as proporções de homens e, principalmente, de mulheres com rendimentos inferiores ao salário mínimo. Ainda assim, as mulheres que ganhavam menos de um salário mínimo, em 2002, eram em proporção maior (14,2\%) do que os homens $(9,7 \%)$. Na faixa de 1 a 3 salários mínimos, encontrava-se a maioria dos trabalhadores $(48,6 \%$ no caso dos homens e $52,3 \%$ para as mulheres). Mas um dos fatos mais interessantes na década de 90 foi o maior aumento na proporção de muIheres com remunerações relativamente altas, o que contribuiu para uma elevação mais acentuada da média dos rendimentos femininos. Medindo o nível de rendimento pelos quartis da distribuição, constatou-se uma evolução positiva nos três quartis dos rendimentos femininos, enquanto para os homens só houve aumento no valor do 1 의 quartil, como conseqüência da mencionada estruturação de ocupações mal remuneradas devido à expansão da participação dos adultos.

Os homens sofreram mais do que as mulheres com as mudanças na estrutura do emprego na década de 90 , quando empregos relativamente bem remunerados foram eliminados e uma elevada proporção dos que surgiram não foi tão bem remunerada. Os efeitos dessas transformações sobre as remunerações dos homens aparecem claramente no declínio do valor da mediana e do 3 o quartil, mas não transparecem no valor do rendimento médio dos homens porque, nos anos 90 , proliferaram os pequenos empregadores, a maioria homens com rendimento relativamente alto em comparação aos salários dos empregados, elevando o valor da média das remunerações do trabalho masculino.

No caso das mulheres, o aumento do valor da média, da mediana e do 3 o quartil da distribuição dos rendimentos teve outra razão, refletindo, principalmente, a ampliação de empregos em atividades sociais que continuam absorvendo uma parcela importante da PEA feminina e possibilitam remunerações relativamente elevadas.

Os avanços das mulheres em termos de elevação de seus rendimentos não foram, contudo, suficientes para eliminar a enorme diferença existente em relação aos homens. Assim, em 2002, os rendimentos masculinos superavam os femininos em torno de $20 \%$, qualquer que fosse o indicador quartílico utilizado. Isso faz parte de um contexto de enorme diferenciação de remunerações, em que se ressaltam as diferenças de escolaridade, destacando-se os altos rendimentos das pessoas com nível superior de instrução que, na década de 90 , se afastaram ainda mais dos rendimentos proporcionados pelos demais níveis de escolaridade.

É importante destacar que a melhoria generalizada da escolaridade das pessoas ocupadas manifestou-se quase que exclusivamente em elevação de rendimentos dos ocupados com curso superior completo, contribuindo para tornar ainda mais acentuadas as diferenças de rendimento entre os trabalhadores com curso superior e os demais, apesar da crescente participação das mulheres com curso superior, que já ultrapassou a metade desses trabalhadores com alta escolaridade.

As diferenças de rendimento entre homens e mulheres, segundo posição na 
ocupação e anos de estudo, diminuíram na década de 90 , mas isso não ocorreu de forma homogênea conforme os níveis de escolaridade. $\mathrm{Na}$ verdade, essa redução reflete, de um lado, ganhos mais acentuados de rendimentos femininos nos níveis de escolaridade superior (15 anos de estudo ou mais) e inferior (menos de oito anos de estudo) e, de outro lado, perdas mais acentuadas de rendimento dos homens nos níveis intermediários de escolaridade (ensinos fundamental e médio completos).

O maior aumento dos rendimentos femininos entre trabalhadores que não completaram o ensino fundamental é conseqüência do elevado peso do emprego doméstico na absorção de mulheres com esse nível de escolaridade, sendo que este tipo de ocupação, por ser muito mal remunerado, teve expressivo aumento de rendimento mediano. Nos níveis intermediários de escolaridade, verificou-se queda maior de rendimentos para homens com ensino fundamental completo e redução bem mais acentuada, sem distinção de sexo, entre os trabalhadores com ensino médio completo (11 a 14 anos de estudo).

Este é um dos fatos mais marcantes do mercado de trabalho na década de 90: trabalhadores de ambos os sexos com nível de instrução relativamente alto (ensino médio completo) auferiam, em 2002, rendimentos não muito maiores do que os obtidos, dez anos antes, por uma população trabalhadora menos instruída, ocorrendo queda substancial do rendimento mediano dos trabalhadores com ensino médio completo, o que indica que a economia, com um desempenho medíocre, não conseguiu aproveitar melhor uma população trabalhadora mais bem instruída.

Quando se comparam níveis de rendimento segundo faixas de escolaridade com uma diferença no tempo de dez anos, é preciso levar em conta que o aumento geral da escolaridade da população modifica a composição etária das pessoas que fazem parte de cada uma das faixas de escolaridade (WAJMANN e MENEZES, 2003). Assim, em uma década, diminuiu a participação dos jovens comparativamente à dos adultos na faixa de menos de oito anos de estudo, ocorrendo o contrário nas demais faixas de escolaridade. Essa mudança de composição etária tende a ressaltar eventuais aumentos do nível de rendimento para os que não têm o ensino fundamental completo, suavizando os aumentos nas demais faixas de escolaridade.

$\mathrm{Na}$ realidade, as mudanças de composição etária reforçaram tanto o aumento observado nos rendimentos dos trabalhadores de ambos os sexos que não têm ensino fundamental completo quanto a redução para aqueles com ensino médio completo. Porém, o expressivo aumento do rendimento mediano dos trabalhadores com nível superior ocorreu apesar das mudanças na composição etária, o que permite ressaltar ainda mais a expansão nos rendimentos dos trabalhadores com nível superior completo. Dessa forma, o comportamento das remunerações do trabalho, na década de 90, contribuiu para tornar ainda mais expressivas as diferenças de rendimento entre os ocupados com curso superior completo e o resto dos trabalhadores. A diferença proporcional de rendimento mediano dos trabalhadores com nível superior de escolaridade comparativamente aos que têm ensino médio completo mais do que duplicou, atingindo, em 2002, 266,7\%, no caso das mulheres, e $243 \%$, para os homens.

O aumento nos níveis de instrução da população brasileira e as maiores exigências de escolaridade por parte dos empregadores resultaram em melhoria do perfil educacional da população ocupada. As mulheres, em particular, ampliaram sua participação relativa em todos os níveis de escolaridade, mas foi no nível superior que mais aumentou a proporção de mulheres ocupadas, atingindo $51 \%$ em 2002. Mas ainda que as mulheres ocupadas constituam mais da metade dos ocupados com nível superior, seus rendimentos continuam extremamente desfavoráveis em relação aos dos homens. Essa desvantagem, ainda que tenha diminuído na última década, continua bastante expressiva em todos os níveis de escolaridade. Contudo, é justamente no nível superior que as 
diferenças de rendimento mediano em favor dos homens são mais expressivas. Neste grau de escolaridade (15 anos e mais), o rendimento mediano masculino, em 2002 , superava o feminino em $47 \%$, o que, em termos absolutos, corresponde a quase 3,5 salários mínimos.

\section{Diferenças de rendimentos do trabalho em 2004}

É possível caracterizar as diferenças de rendimentos do trabalho no Brasil metropolitano, em 2004, considerando, de forma conjunta, os rendimentos medianos das pessoas por posição na ocupação e nível de escolaridade. Assim, podem-se identificar quatro grupos básicos de trabalhadores (Tabela 1), classificados conforme os rendimentos, em ordem ascendente:

- o Grupo D, representativo das remunerações muito baixas, é formado pelo trabalho doméstico remunerado, pelos empregados de estabelecimentos sem carteira de trabalho assinada e com menos de dez anos de estudo e pelos trabalhadores por conta-própria com menos de oito anos de estudo (rendimento mediano menor que 1,5 salário mínimo);

- o Grupo C, do trabalhador "típico" brasileiro, é constituído por todos os empregados com vínculo formalizado e grau de instrução inferior ao ensino médio, pelos trabalhadores por conta-própria com ensino fundamental completo e pelos empregados sem carteira que têm ensino médio completo, porém não completaram nível superior;

- o Grupo B, de rendimentos medianos de 3 a 4 salários mínimos (no caso dos homens), inclui os empregados formais e trabalhadores por conta-própria com ensino médio completo e os empregadores que não possuem ensino médio;

- o Grupo A, de rendimentos ainda mais elevados (mediana superior a cinco salários mínimos, no caso dos homens), engloba empregados formais e não-formais, trabalhadores por conta-própria e empregadores de nível superior, além dos empregadores com ensino médio completo.

A diferença de nível de rendimento entre homens e mulheres pode ser associada às diferentes distribuições por sexo, segundo as categorias de ocupação (que descrevem as diferenças de rendimento) e às diferenças de rendimento entre sexos, dentro de cada uma dessas categorias.

No grupo de rendimentos muito baixos, as diferenças por sexo são relativamente pequenas (menos de 10\% no caso da mediana, com exceção do trabalho por conta-própria). Este grupo responde por cerca de $25 \%$ na absorção de ocupados de ambos os sexos. Ou seja, $1 / 4$ de homens e mulheres têm ocupações de rendimentos muito baixos e as diferenças entre eles são relativamente pequenas (Tabela 2 ).

No grupo de trabalhadores "típicos", as diferenças de rendimentos por sexo são de aproximadamente $20 \%$, que é a diferença padrão de rendimentos por sexo

TABELA 1

Distribuição dos ocupados, por sexo, segundo grupos de trabalhadores definidos pelo nível de rendimento Regióes Metropolitanas Selecionadas (1) - 2004

\begin{tabular}{lcc}
\hline Grupos de Trabalhadores & Homens & Mulheres \\
\hline Total & $\mathbf{1 0 0 , 0}$ & $\mathbf{1 0 0 , 0}$ \\
D & 22,8 & 25,0 \\
C & 33,4 & 5,4 \\
B & 29,4 & 21,1 \\
A & 14,4 & 29,9 \\
\hline
\end{tabular}

Fonte: IBGE. Pesquisa Nacional por Amostra de Domicílios - PNAD.

(1) Referem-se às Regiões Metropolitanas de Belém, Fortaleza, Recife, Salvador, Rio de Janeiro, Belo Horizonte, Curitiba, Porto Alegre, São Paulo e Distrito Federal. 
correspondente às diferenças de medianas de rendimentos de homens e mulheres de toda a população ocupada nas regiões metropolitanas. Esse grupo abrange 33\% dos homens ocupados e somente $5 \%$ das mulheres.

A participação da mulher é maior no conjunto dos outros dois grupos de rendimentos relativamente altos, principalmente no mais elevado. Nesses dois grupos, entretanto, as diferenças de rendimentos em favor dos homens são muito grandes.

As diferenças de rendimento, por sexo, são pequenas no trabalho doméstico e entre os empregados sem carteira assinada e com escolaridade inferior ao ensino médio (menos de $10 \%$ no caso do rendimento mediano). A diferença padrão de rendimentos por sexo (20\%) é característica do emprego formal com nível de instrução inferior ao ensino médio e do emprego sem carteira com ensino médio ou mais, enquanto a diferença é maior (mais de $30 \%$ no caso da mediana) para o emprego formal de nível superior de escolaridade e para os trabalhadores por conta-própria e empregadores de qualquer nível de instrução.

No Brasil, as pessoas com nível superior de escolaridade têm rendimentos muito maiores do que as outras ocupadas em todas as posições na ocupação. Dessa forma, embora as diferenças de rendimento por sexo sejam muito grandes em todos os níveis de instrução, no caso dos empregadores e trabalhadores por conta-própria, as maiores diferenças absolutas por gênero se verificam no grau superior. Por esse motivo, é importante analisar detalhadamente as diferenças de rendimentos entre os ocupados com este nível de escolaridade.

\section{Diferencas de rendimentos do trabalho entre homens e mulheres com nível superior de instrução em 2004}

A diferença de rendimentos medianos entre homens e mulheres com nível superior de instrução é de $40 \%$ (9,4 salários

TABELA 2

Relação de rendimentos masculinos e femininos, segundo grupos de trabalhadores definidos pelo nível de rendimento, posição na ocupação e anos de estudo Regiões Metropolitanas Selecionadas (1) - 2004

\begin{tabular}{|c|c|c|}
\hline Grupos & Posição na ocupação e anos de estudo & $\begin{array}{c}\text { Rendimentos medianos } \\
\text { homem/mulher }\end{array}$ \\
\hline \multirow{4}{*}{ D } & Trabalho doméstico & 0,946 \\
\hline & Emprego não-formal, menos de 8 anos de estudo & 1,095 \\
\hline & Emprego não-formal, de 8 a 10 anos de estudo & 1,039 \\
\hline & Conta-própria, menos de 8 anos de estudo & 1,393 \\
\hline \multirow{4}{*}{$\mathbf{C}$} & Emprego formal, menos de 8 anos de estudo & 1,203 \\
\hline & Emprego formal, de 8 a 10 anos de estudo & 1,223 \\
\hline & Conta-própria, de 8 a 10 anos de estudo & 1,371 \\
\hline & Não-formal, de 11 a 14 anos de estudo & 1,229 \\
\hline \multirow{4}{*}{ B } & Empregador, menos de 8 anos de estudo & 1,257 \\
\hline & Empregador, de 8 a 10 anos de estudo & 1,452 \\
\hline & Conta-própria, de 11 a 14 anos de estudo & 1,500 \\
\hline & Emprego formal, de 11 a 14 anos de estudo & 1,274 \\
\hline \multirow{5}{*}{ A } & Empregador, de 11 a 14 anos de estudo & 1,338 \\
\hline & Empregador, mais de 15 anos de estudo & 1,287 \\
\hline & Emprego não-formal, mais de 15 anos de estudo & 1,200 \\
\hline & Conta-própria, mais de 15 anos de estudo & 1,333 \\
\hline & Emprego formal, mais de 15 anos de estudo & 1,481 \\
\hline
\end{tabular}

Fonte: IBGE. Pesquisa Nacional por Amostra de Domicílios - PNAD.

(1) Referem-se às Regiões Metropolitanas de Belém, Fortaleza, Recife, Salvador, Rio de Janeiro, Belo Horizonte, Curitiba, Porto Alegre, São Paulo e Distrito Federal. 
mínimos para homens e 6,7 para mulheres). ${ }^{2}$ Há uma grande diferença por posição na ocupação, destacando-se nos extremos o rendimento elevado dos empregadores e o relativamente baixo dos empregados sem carteira (1:2,4 no caso dos homens e 1 : 2,2 para as mulheres). O peso do emprego não-formal na absorção do pessoal de nível superior é semelhante para homens e mulheres (13\% a $14 \%)$, enquanto os empregadores têm maior participação na absorção de homens (12\%) do que de mulheres (5\%). Os rendimentos medianos do emprego formal e do trabalho por contaprópria situam-se em nível intermediário, entre os rendimentos dos empregados sem carteira e dos empregadores ( 9 a 10 salários mínimos para homens e 6,5 a 7 salários para mulheres). 0 trabalho por conta-própria possui maior participação na absorção de homens do que de mulheres de nível superior (17\% e 13\%, respectivamente), ocorrendo o contrário com o emprego formal (58\% e $68 \%$, respectivamente) (Tabela 3)

Os diferentes perfis por posição na ocupação de homens e mulheres com nível superior de instrução ressaltam as diferenças de rendimento por sexo, mas as diferenças dentro de cada posição na ocupação são muito grandes (salvo entre empregados sem carteira de trabalho). Neste particular, destaca-se a enorme diferença de rendimentos por sexo no emprego formalizado, posição na ocupação responsável pela maior parte da absorção de homens e mulheres com alto grau de instrução.

A explicitação do tipo de ocupação permite destacar a importância do diploma de nível superior para o acesso aos cargos bem remunerados. Pessoas com mais de 15 anos de estudo, mas em ocupações de serviço administrativo ou trabalho manual, mesmo quando trabalhador por contaprópria e não somente empregado com ou sem vínculo formalizado, têm remuneração mediana muito menor do que a do empregador (todos eles declaram-se dirigentes ou profissionais de nível superior), bem como de empregados e trabalhadores por conta-própria, dirigentes, profissionais de nível superior e técnicos de nível médio. Assim, remunerações medianas acima de 11 salários mínimos, para homens, e de 7,5 salários mínimos, para mulheres, são verificadas somente para empregadores, empregados formais dirigentes e profissionais de nível superior, empregados sem carteira dirigentes e conta-própria profissionais de nível superior. Os rendimentos medianos situam-se entre 8 e 11 salários mínimos para homens e de 5,5 a 7 salários mínimos para mulheres, no caso do conta-própria e empregado formal, técnicos de nível médio e empregados sem carteira profissional de nível superior. Os salários são bem mais baixos (no máximo 4,5 salários mínimos)

TABELA 3

Distribuição dos ocupados com curso superior e rendimentos medianos, por sexo, segundo posição na ocupação Regiões Metropolitanas Selecionadas (1) - 2004

\begin{tabular}{|c|c|c|c|c|c|}
\hline \multirow{2}{*}{$\begin{array}{c}\text { Posição na } \\
\text { Ocupação }\end{array}$} & \multicolumn{2}{|c|}{ Homens } & \multicolumn{2}{|c|}{ Mulheres } & \multirow{2}{*}{ Homem/Mulher } \\
\hline & Ocupados (\%) & Mediana (em SM) & Ocupados (\%) & Mediana (em SM) & \\
\hline Total & 100,0 & 9,36 & 100,0 & 6,69 & 1,399 \\
\hline Emprego formal & 58,1 & 10,13 & 68,4 & 6,69 & 1,514 \\
\hline Emprego não-formal & 13,3 & 5,57 & 13,9 & 4,70 & 1,185 \\
\hline Conta-própria & 16,7 & 9,17 & 12,5 & 6,69 & 1,371 \\
\hline Empregador & 11,9 & 13,38 & 5,2 & 10,13 & 1,321 \\
\hline
\end{tabular}

Fonte: IBGE. Pesquisa Nacional por Amostra de Domicílios - PNAD.

(1) Referem-se às Regiões Metropolitanas de Belém, Fortaleza, Recife, Salvador, Rio de Janeiro, Belo Horizonte, Curitiba, Porto Alegre, São Paulo e Distrito Federal.

${ }^{2}$ Os rendimentos foram expressos em salários mínimos/hora. O salário mínimo hora em 2004 era $\mathrm{R} \$ 1,38$ (considerou-se no cálculo a jornada semanal de 44 horas e 4,28 semanas por mês, conforme a legislação existente). 
para empregados sem carteira técnicos, conta-própria e empregado formal em ocupações de trabalho manual e empregado formal e não-formal do serviço administrativo. As diferenças de rendimento, então, são grandes e dependem do fato de ser o dono do negócio ou simples empregado, do cargo ocupado, do tamanho do estabelecimento empregador e da formalização ou não do vínculo de emprego. O simples fato de ter mais de 15 anos de estudo não garante o elevado rendimento, a menos que se tenha efetivamente ingressado no cargo bem remunerado que exige diploma de nível superior (Tabela 4).

As distribuições de homens e mulheres com nível superior de instrução, por tipo e posição na ocupação, são muito diferentes: os homens têm maior participação entre os empregadores, empregados formais dirigentes, trabalhadores por conta-própria e empregados formais com ocupação de trabalho manual, enquanto as mulheres participam mais entre os empregados formais e não-formais profissionais de nível superior. Os homens com nível superior de instrução estão mais presentes entre empregadores e dirigentes e as mulheres predominam entre os empregados profissionais de nível superior. Entre o pessoal com curso superior, os homens são $2 / 3$ dos empregadores e $55 \%$ dos empregados com cargo de direção. Já as mulheres representam $61 \%$ dos empregados profissionais de nível superior.

As diferenças de rendimento mediano por sexo, embora sejam grandes em todas as categorias de tipo e posição na ocupação, são particularmente elevadas entre empregados formais e não-formais dirigentes, empregados formais e nãoformais profissionais de nível superior, conta-própria técnico e empregado nãoformal de serviço administrativo. A enorme diferença de rendimento por sexo no emprego em cargo de direção ou em ocupação profissional de nível superior sugere que as mulheres com ensino superior têm nessas ocupações acesso predominante a cargos pior remunerados. Em grande medida, entretanto, essa diferença está associada aos setores de

TABELA 4

Distribuição dos ocupados com nível superior de escolaridade e rendimentos medianos, por sexo, segundo tipo e posição na ocupação Regiões Metropolitanas Selecionadas (1) - 2004

\begin{tabular}{|c|c|c|c|c|c|}
\hline \multirow{2}{*}{ Tipo e posição na ocupação } & \multicolumn{2}{|c|}{ Homens } & \multicolumn{2}{|c|}{ Mulheres } & \multirow{2}{*}{$\begin{array}{c}\text { Mediana } \\
\text { H/M }\end{array}$} \\
\hline & $\begin{array}{c}\text { Ocupados } \\
\text { (\%) }\end{array}$ & $\begin{array}{l}\text { Mediana } \\
\text { em (SM) }\end{array}$ & $\begin{array}{c}\text { Ocupados } \\
\text { (\%) }\end{array}$ & $\begin{array}{l}\text { Mediana } \\
\text { (em SM) }\end{array}$ & \\
\hline Total & 100,0 & 9,36 & 100,0 & 6,69 & 1,399 \\
\hline Empregador & 11,9 & 13,38 & 5,2 & 10,13 & 1,321 \\
\hline Empregado formal dirigente & 9,4 & 13,38 & 7,4 & 8,92 & 1,500 \\
\hline Empregado formal profissional nível superior & 23,4 & 12,74 & 35,9 & 8,03 & 1,587 \\
\hline Conta-própria profissional nível superior & 7,5 & 11,89 & 7,0 & 8,92 & 1,333 \\
\hline Empregado não-formal dirigente & 1,8 & 11,15 & 1,4 & 7,58 & 1,471 \\
\hline Conta-própria técnico & 3,5 & 10,70 & 1,9 & 6,32 & 1,693 \\
\hline Empregado formal técnico & 9,2 & 8,92 & 8,9 & 6,67 & 1,337 \\
\hline Emp. não-formal profissional nível superior & 5,4 & 8,03 & 7,0 & 5,49 & 1,463 \\
\hline Empregado não-formal técnico & 2,3 & 4,46 & 2,0 & 4,46 & 1,000 \\
\hline Conta-própria trabalho manual & 1,9 & 4,28 & - & - & - \\
\hline Empregado formal serviço administrativo & 2,0 & 4,12 & 1,7 & 2,27 & 1,815 \\
\hline Empregado formal trabalho manual & 3,5 & 4,05 & - & - & - \\
\hline Empregado não-formal serviço administrativo & 1,6 & 3,96 & 2,4 & 2,68 & 1,478 \\
\hline
\end{tabular}

Fonte: IBGE. Pesquisa Nacional por Amostra de Domicílios - PNAD.

(1) Referem-se às Regiões Metropolitanas de Belém, Fortaleza, Recife, Salvador, Rio de Janeiro, Belo Horizonte, Curitiba, Porto Alegre, São Paulo e Distrito Federal. 
atividade em que se ocupam os homens e mulheres com instrução de nível superior (Tabela 5).

De fato, a distribuição setorial dos empregados com nível superior de instrução varia entre os sexos. As mulheres estão mais presentes nas atividades de educação, saúde e serviços sociais, enquanto os homens apresentam maior variedade setorial de empregos. Assim a participação das mulheres com nível superior de instrução é de somente 1/4 na construção civil, 1/3 na indústria de transformação, outras atividades industriais (serviços de utilidade pública) e transporte, armazenagem e comunicação, 1/2 no comércio e reparação, administração pública, serviços domésticos e outras atividades (finanças e imóveis), 2/3 em alojamento e alimentação e 3/4 na educação, saúde e serviços sociais.

Assim, as mulheres com nível superior de escolaridade se ocupam principalmente em empregos da administração pública, educação, saúde, serviços sociais e atividades financeiras (77\% do emprego de mulheres de nível superior de instrução), enquanto os homens estão mais repartidos por outras atividades, já que as mesmas mencionadas para as mulheres abarcam somente $58 \%$ dos empregados masculinos com nível superior de instrução. Mesmo nos setores em que predomina o emprego de mulheres, as diferenças de rendimento em favor dos homens são muito grandes. No caso dos empregos formalizados, essa diferença é de $55,5 \%$ em educação, saúde e serviços sociais, 33,3\% em finanças e imóveis e 21,8\% em administração pública. Esses dados sugerem que os homens têm acesso a cargos mais bem remunerados de direção e de ocupações profissionais de nível superior.

\section{Conclusão}

No Brasil, as remunerações relativamente altas das pessoas ocupadas com nível superior de instrução têm importância muito grande na enorme desigualdade dos rendimentos do trabalho. As transformações na economia e o estreitamento do mercado de trabalho, ao longo dos anos 90 , não diminuíram essa importância das diferenças de rendimento das pessoas com elevada escolaridade. $O$ contraste é imenso entre o que aconteceu com os rendimentos das pessoas com escolaridade média e superior. O fraco desempenho da economia e o estreitamento do mercado de trabalho desvalorizaram fortemente o trabalho das

TABELA 5

Distribuição dos ocupados com nível superior de instrução, por sexo, segundo setor de atividade econômica Regiões Metropolitanas Selecionadas (1) - 2004

\begin{tabular}{lccc}
\multicolumn{1}{c}{ Setores de atividade econômica } & Homens & Mulheres & $\begin{array}{c}\text { Em porcentagem } \\
\text { Proporção de } \\
\text { Mulheres }\end{array}$ \\
\hline Total & 100,0 & $\mathbf{1 0 0 , 0}$ & $\mathbf{5 4 , 7}$ \\
Agrícola & 0,3 & - & - \\
Indústria de Transformação & 2,0 & 0,8 & 32,1 \\
Outra atividade industrial & 16,1 & 6,8 & 33,6 \\
Construção & 3,2 & 0,9 & 25,1 \\
Comércio e reparação & 8,2 & 6,1 & 47,4 \\
Alojamento e alimentação & 0,7 & 0,9 & 63,4 \\
Transporte, armazenagem e comunicação & 7,0 & 3,3 & 36,4 \\
Administração pública & 17,4 & 14,8 & 50,6 \\
Educação, saúde, serviços sociais & 18,8 & 45,2 & 74,3 \\
Outros serviços coletivos, sociais, pessoais & - & 0,0 & - \\
Serviço doméstico & 4,6 & 4,2 & 52,0 \\
Outras atividades & 21,3 & 16,9 & 48,8 \\
Resíduo & 0,3 & 0,1 & - \\
\hline
\end{tabular}

Fonte: IBGE. Pesquisa Nacional por Amostra de Domicílios - PNAD.

(1) Referem-se às Regiões Metropolitanas de Belém, Fortaleza, Recife, Salvador, Rio de Janeiro, Belo Horizonte, Curitiba, Porto Alegre, São Paulo e Distrito Federal. 
pessoas com nível médio de instrução, não ocorrendo o mesmo para aquelas com nível superior. Este último grupo apresentou significativas mudanças de composição, aumentando a participação relativa das mulheres e aproximando o nível relativo das remunerações por sexo, com as mulheres sustentando mais do que os homens o poder de compra de suas remunerações. $O$ aumento da participação relativa das mulheres no grupo de trabalhadores de nível superior de instrução está relacionado ao tipo de ocupação dessas mulheres, destacando-se o emprego em atividades de educação, saúde, serviço social, finanças e administração pública. Salvo no setor de finanças, nas demais atividades que abarcam o grosso do emprego feminino de nível superior de escolaridade, o mercado de trabalho foi menos afetado desfavoravelmente pelas mudanças na economia durante a década de 90.

As diferenças de rendimento por sexo são muito grandes entre os empregadores e trabalhadores por conta-própria em todos os níveis de instrução e entre os empregados assalariados de nível médio e, principalmen-

\section{Referências bibliográficas}

BALTAR, P. Estrutura econômica e emprego urbano na década de 1990. In: PRONI, M. W.; HENRIQUE, W. (Orgs.). Trabalho, mercado e sociedade: o Brasil nos anos 90. São Paulo: Editora Unesp; Campinas: Instituto de Economia da Unicamp, 2003.

BALTAR, P. Desigualdade de rendimentos do trabalho e salário mínimo na década de 90. Carta Social e do Trabalho, Campinas, Unicamp/IE/Cesit, n. 1, maio a agosto de 2005. Disponível em: <http://www.unicamp.br>. Acesso em: 1 mar. 2006.

BELTRÃO, K. I.; ALVES, J. E. D. A reversão do hiato de gênero na educação brasileira no século XX. In: ENCONTRO NACIONAL DE ESTUDOS POPULACIONAIS, 14, Caxambu, 2004. Anais... Belo Horizonte: Abep, 2004.

CARRASCO, C. et al. Mujeres, trabajos y políticas sociales: una aproximación al te, entre os que têm o nível superior. Considerando somente o pessoal de nível superior, o perfil por posição na ocupação de homens e mulheres é diferente, com a participação dos não-assalariados (empregadores e trabalhadores por conta-própria) maior entre os homens. A diferença de rendimento por sexo entre esses não-assalariados é muito grande, mas é ainda maior no emprego formalizado, justamente a posição que pesa mais na ocupação de mulheres com nível superior de instrução.

Do ponto de vista das diferenças de rendimento por sexo, é uma vantagem para os homens sua maior presença entre os não-assalariados, mas o que chama atenção é a acentuada diferença de rendimento por sexo entre os empregados formais, em todos os tipos de postos de trabalho: cargos de direção, ocupações profissionais de nível superior, ocupações técnicas e de serviços administrativos. Essas diferenças diminuíram na década de 90 , mas ainda continuam significativas. As mulheres avançaram em termos de acesso aos cargos mais bem remunerados, porém, nesses cargos, ainda predomina a presença masculina.

caso español. Colección Estúdios, Madrid, Instituto de la Mujer, № 51,1998.

Guedes, M. de C.; ALVES, J. E. D. A população feminina no mercado de trabalho entre 1970-2000: particularidades do grupo com nível universitário. In: ENCONTRO NACIONAL DE ESTUDOS POPULACIONAIS, 14, Caxambu, 2004. Anais... Belo Horizonte: Abep, 2004.

HIRATA, H. Entrevista. 1 CADERNO Globalização. Disponível em: < http:// www2.correioweb.com.br/cw/2001-1-28/ mat 25423.htm>. Acesso em: 5 mar. 2006.

LEONE, E.; BALTAR, P. Mercado de trabalho metropolitano: gênero e diferenças de rendimento, 1992-2002. In: Encontro Nacional de Estudos do Trabalho, 9, Recife, 2005. Anais... Recife: Abet, 2005.

LAVINAS, L. Evolução do desemprego feminino nas áreas metropolitanas. In: 
ROCHA, M. I. B. (Org.). Trabalho e gênero: mudanças, permanências e desafios. Campinas: Abep, Nepo/Unicamp e Cedeplar/ UFMG. São Paulo: Editora 34, 2000.

Emprego feminino no Brasil: mudanças institucionais e novas inserções no mercado de trabalho. In: LAVINAS; LEÓN (Coords.). Serie Políticas Sociais n: 60. Santiago de Chile: Cepal, setembro de 2002.

MELO, H. Trabalhadoras domésticas: o eterno lugar feminino. Uma análise dos grupos ocupacionais. OIT Brasil/Ipea, 1999.

MONTALI, L. Rearranjos familiares de inserção, precarização do trabalho e empobrecimento. In: ENCONTRO NACIONAL DE ESTUDOS POPULACIONAIS, 14, Caxambu, 2004. Anais... Belo Horizonte: Abep, 2004.
VARELLA, M. R. La política de igualdad en el ámbito del trabajo: una propuesta de construcción teórica desde el género. In: RADL, P. R. Cuestiones actuales de sociologia del gênero. Espanha: Universidad de Santiago de Compostela, 2001.

WAJMANN, S.; MENEZES, N. Os efeitos da mudança demográfica sobre a desigualdade de rendimentos no Brasil. In: WAJMANN; MACHADO (Orgs.). Mercado de trabalho: uma análise a partir das pesquisas domiciliares no Brasil. Belo Horizonte: Editora UFMG, 2003.

WAJMANN, S.; RIOS NETO, E. Quantas serão as mulheres: cenários para a atividade feminina. In: ROCHA, M. I.; BALTAR (Orgs.). Trabalho e gênero: mudanças, permanência e desafios. Abep, Nepo/Unicamp e Cedeplar/UFMG. Editora 34, 2000.

\section{Abstract \\ Differences in income from labor between men and women with college education in large cities}

This article presents an analysis of the difference in income between working men and women with college degrees in the various metropolitan regions in Brazil. The study is based on two factors: a) although the difference between men's and women's incomes has fallen over the last decade, they are nevertheless still considerable, and are part of the context of enormous variations in income, where the differences in schooling carry considerable weight. In this respect, college-educated persons earn considerably more, and the difference between such incomes and those earned by persons with lower educational levels have become even greater over the last decade; b) the greatest differences in income between men and women are found among persons with college degrees, which is exactly the bracket where women show the greatest presence. This article analyzes the changes that occurred during the 1990s and the levels and dispersion of incomes of working persons, and shows differences according to gender, educational level and position in the occupation. The article also refers to a specific study, regarding the year 2004, on the differences in income between men and women with college degrees, according to position in the occupation and sector of activity. The type of occupation is also considered (managers in general, professionals in the arts and sciences, high-school level technicians, workers in administrative services, and other occupations) are similar to the hierarchy of positions. The source of data is the National Household Survey by Sample (PNAD/IBGE).

Key words: Labor market. Income. Gender. Schooling.

Recebido para publicação em 29/09/2006. Aceito para publicação em 06/11/2006. 
